\title{
A Avaliação do Desenvolvimento Infantil: um Desafio Interdisciplinar
}

\author{
Evaluation of Childhood Development: an \\ Interdisciplinary Challenge
}

Cristina Gonçalves Alvim I', Fabiano Gonçalves Guimarães ${ }^{I I}$, Niriana Lara Santos Meinberğ Larissa Tavares AguiarI, Livia Cristina Guimarães CaetanoI, Loyane Cabral CarruscaI, Luciana Machado CaetanoI, Ludimila LabancaI , Nathália de Magalhães FonsecaI, Rafael Antônio Madeira PauloI, Thaysa Leite Tagliaferri ${ }^{I}$

\author{
PALAVRAS-CHAVE: \\ - Desenvolvimento Infantil; \\ - Saúde da Criança; \\ - Educação Médica; \\ - Atenção Integrada às Doenças \\ Prevalentes na Infância.
}

\author{
KEYWORDS: \\ - Child Development; \\ - Child Health; \\ - Medical Education; \\ - Integrated Management of \\ Childhood Illness.
}

Recebido em: 30/03/2010

Aprovado em: 19/11/2010

REVISTA BRASILEIRA DE EDUCAÇÃO MÉDICA $5 1 \longdiv { 3 6 ( 1 , \text { Supl. 1): } 5 1 - 5 6 2 0 1 2 }$

\section{RESUMO}

OBJETIVO: Avaliar o desenvolvimento de crianças de 2 meses a 2 anos de idade por meio da Atenção Integrada às Doenças Prevalentes na Infância (AIDPI), no contexto do Programa de Educação pelo Trabalho em Saúde (PET-Saúde). MÉTODO: Estudo transversal realizado com 122 crianças, com idades entre 2 meses e 2 anos, da área de abrangência do Centro de Saúde São Bernardo (CSSB) - Belo Horizonte (MG), em 2009. Os dados relativos ao desenvolvimento foram obtidos através da aplicação de dois questionários: AIDPI e Caderneta de Saúde da Criança (CSC). Foram comparadas as classificações do desenvolvimento pela AIDPI e pela CSC, a associação entre atraso do desenvolvimento e as variáveis estudadas. RESULTADOS: As características com maior frequência na população estudada foram a baixa escolaridade das mães (62,1\%), seguida de parentes com deficiência mental (71,3\%) e problemas na gestação (71,3\%). A AIDPI evidenciou que $61,5 \%$ da população estudada encontra-se normal com fator de risco, 16,4\% normal sem fator de risco, 11,5\% com possível atraso e 10,7\% com provável atraso do desenvolvimento infantil. A concordância observada entre a classificação da AIDPI e da CSC foi de 0,34, coeficiente Kappa igual a - 0,12 $(p=0,98)$. Não houve associação estatisticamente significativa entre as variáveis analisadas (frequenta creches; convívio com problemas emocionais; escolaridade da mãe; idade gestacional; e peso ao nascer) e atraso possível/provável do desenvolvimento identificado pela AIDPI. CONCLUSÃO: O PET-Saúde, como proposta de integração da educação pelo trabalho, permitiu uma oportunidade de convivência e troca de experiências entre alunos e profissionais de diferentes áreas de atuação, trabalhando em um projeto comum.

\begin{abstract}
OBJECTIVE: The aim of this study was to evaluate early childhood development, from two months to two years of age, based on Integrated Management of Childhood Illness, IMCI (or AIDPI in Portuguese) in the context of the Educational Program for Health Work (PET-Saúde). METHODS: A cross-sectional study was conducted with 122 children two months to two years of age from the coverage area of the São Bernardo Health Center in Belo Horizonte, Minas Gerais State, in 2009. Data on the children's development were obtained using two questionnaires: IMCI and the Children's Health Booklet (CSC in Portuguese). Early childhood development according to the IMCI classification was compared to the results with CSC. The authors also investigated potential associations between independent variables and developmental delay. RESULTS: The most frequent characteristics in the study population were low maternal schooling $(62.1 \%)$, followed by relatives with mental disability (71.3\%), and problems during the pregnancy $(71.3 \%)$. Based on the IMCI classification, $61.5 \%$ of the study population was normal with some risk factor, $16.4 \%$ normal without any risk factor, $11.5 \%$ with possible delay, and 10.7\% with probable childhood developmental delay. Agreement between the IMCI and CSC classifications was 0.34, kappa index-0.12 ( $p=0.98)$. There was no statistically significant association between the variables (daycare attendance; contact with emotional problems; maternal schooling; gestational age; and birth weight) and possible or probable early childhood developmental delay according to the IMCI classification. CONCLUSION: PET-Saúde, as a proposal for integration between education and work, provided an opportunity for contact and exchange of experiences between students and health professionals from various fields, working in a common project.
\end{abstract}

I Universidade Federal de Minas Gerais, Belo Horizonte, MG, Brasil.

"Prefeitura Municipal de Belo Horizonte, Belo Horizonte, MG, Brasil; Universidade Federal de Minas Gerais, Belo Horizonte, MG, Brasil.

III Prefeitura Municipal de Belo Horizonte, Belo Horizonte, MG, Brasil. 


\section{INTRODUÇÃO}

O Programa de Educação pelo Trabalho para a Saúde - PET-Saúde - foi criado pela parceria entre o Ministério da Educação, o Fundo Nacional de Saúde, os Conselhos Nacionais de Secretários de Saúde e Instituições Federais de Ensino Superior, objetivando a aprendizagem tutorial no âmbito da Estratégia Saúde da Família e a qualificação em serviço dos profissionais da saúde, além da iniciação ao trabalho dos estudantes das graduações em saúde 1 .

A Universidade Federal de Minas Gerais (UFMG), em parceria com a Secretaria Municipal de Saúde de Belo Horizonte (SMS-BH), realiza um dos 84 projetos selecionados de 66 Instituições de Ensino Superior em 2009. Neste projeto, foram envolvidos graduandos de Educação Física, Enfermagem, Farmácia, Fisioterapia, Fonoaudiologia, Medicina, Medicina Veterinária, Nutrição, Odontologia e Terapia Ocupacional, com a proposta de integrar alunos das diferentes áreas da saúde na Atenção Primária e contribuir para a formação acadêmica deles, primariamente voltada para o ensino teórico e atendimento especializado individual ${ }^{1}$.

Bispo Júnior aponta que "a universidade tem destacada responsabilidade na condução da formação profissional voltada para a resolução dos problemas e necessidades sociais, e não apenas para o atendimento às regras estabelecidas pelo mercado privatista" ${ }^{\prime 2}$.

O PET-Saúde da UFMG/SMS-BH apresenta três linhas de pesquisa: avaliação das linhas de cuidado por ciclo de vida (saúde da criança, da mulher e do idoso); interface saúde e ambiente; e promoção de modos saudáveis de vida. O objeto desta pesquisa se concentrou na linha de cuidado saúde da criança e avaliação e promoção do cuidado integral, no que se refere ao acompanhamento do desenvolvimento ${ }^{3}$.

O desenvolvimento infantil é uma área que concerne a todos os profissionais da saúde. Esse trabalho em equipe permite um desempenho mais eficiente da intervenção terapêutica ${ }^{4}$. Monteiro afirma que é de extrema importância que todas as ações voltadas para a assistência à saúde sejam desenvolvidas com características de intercâmbio coeso e coerente dos profissionais envolvidos, devendo ser interdisciplinar ${ }^{5}$.

É na infância que a criança adquire as habilidades necessárias para as etapas posteriores do desenvolvimento. Quando este é satisfatório, contribui para a formação de um sujeito com suas potencialidades desenvolvidas, já que os primeiros anos de vida são considerados um período de grande oportunidade para o crescimento e o desenvolvimento sendo, também, um período de vulnerabilidade ${ }^{6,7}$.

Os domínios do desenvolvimento são compostos por quatro grandes áreas: motora; linguagem; adaptativa ou cogniti- va; e social ou pessoal ${ }^{8}$. $\mathrm{O}$ acompanhamento de crianças por diversos profissionais da saúde permite um diagnóstico precoce de alterações ou atrasos no desenvolvimento em alguma dessas áreas. A intervenção precoce irá promover um maior sucesso no tratamento ${ }^{6}$

Para a avaliação do desenvolvimento infantil pode-se utilizar o instrumento da Atenção Integrada às Doenças Prevalentes na Infância (AIDPI), que permite uma linguagem comum entre os profissionais da área de saúde e possibilita o retorno mais efetivo aos pais e/ou cuidadores ${ }^{6}$. A AIDPI, proposta pela Organização Pan-Americana de Saúde (OPAS), tem-se revelado uma estratégia que facilita o acesso a um conjunto de intervenções baseadas em evidência científica e que permitem prevenir e tratar eficazmente os principais problemas de saúde em crianças ${ }^{9}$.

Contrapondo ao modelo tradicional de atenção à saúde, focado na queixa motivadora do atendimento, o uso de questionário padronizado, dados antropométricos e marcos desenvolvimentais presentes na AIDPI possibilitam ações preventivas, avaliação ambiental e promoção de saúde da criança. Sendo assim, o objetivo do presente estudo foi avaliar o desenvolvimento de crianças de 2 meses a 2 anos de idade pela AIDPI ${ }^{9}$.

\section{METODOLOGIA}

Estudo transversal desenvolvido no Centro de Saúde São Bernardo (CSSB), situado na região Norte de Belo Horizonte - MG. A equipe de pesquisadores foi composta por um professor da UFMG (tutor), três profissionais da SMS-PBH (preceptores), seis acadêmicos bolsistas e seis acadêmicos voluntários da UFMG.

Durante o período de agosto a novembro de 2009, foram feitas avaliações do desenvolvimento infantil de crianças de 2 meses a 2 anos de idade residentes na área de abrangência do CSSB cujos pais/responsáveis concordaram em participar da pesquisa. Cada uma das três Equipes de Saúde da Família (ESF) fez o levantamento das crianças na faixa etária definida para o estudo, a partir de cadastros e de informações dos Agentes Comunitários de Saúde (ACS).

Foram excluídas as crianças portadoras de doenças neurológicas previamente diagnosticadas e que causam comprometimento do desenvolvimento neuropsicomotor (DNPM). Crianças que apresentaram algum problema de saúde no dia da avaliação foram reagendadas. Crianças prematuras foram avaliadas segundo a sua idade gestacional corrigida.

Os dados relativos ao desenvolvimento foram obtidos através da aplicação de dois questionários: AIDPI e Caderneta de Saúde da Criança (CSC). 
A AIDPI é um instrumento amplo, porém de fácil aplicação, que operacionaliza e sistematiza a avaliação do DNPM, utiliza marcos do desenvolvimento já validados e acopla a classificação do desenvolvimento a uma conduta. Dessa forma, ela não consiste em um teste diagnóstico e, sim, em um instrumento simples de fácil aplicação. Tem como objetivo sistematizar o atendimento, facilitando, para os profissionais de saúde, a orientação dos pais sobre a promoção do desenvolvimento típico de seus filhos e a detecção precoce de crianças com possibilidades de apresentarem algum problema no desenvolvimento ${ }^{6}$.

A Caderneta de Saúde da Criança (CSC), versão 2007, apresenta quatro marcos do desenvolvimento neuropsicomotor (DNPM) para cada faixa etária até os 10 anos de idade. Ela foi construída com base na escala de Denver II, porém não apresenta uma proposta de classificação e conduta de avaliação de atraso de desenvolvimento ${ }^{10}$.

A coleta dos dados foi realizada no próprio CSSB em dias de vacinação infantil e de consultas pediátricas de rotina ou agendadas pelos ACSs. Foram realizadas também avaliações em visitas domiciliares e nas creches situadas na área de abrangência da CSSB. Foram realizados mutirões nos sábados de campanha de vacinação infantil, cujo objetivo foi captar maior número de crianças para avaliação. Participaram da avaliação das crianças: estudantes de Medicina, Fonoaudiologia, Terapia Ocupacional, Fisioterapia, Educação Física e Farmácia, todos diretamente supervisionados pelos preceptores e tutor. Antes do início da pesquisa, foram realizadas reuniões para apresentação e discussão da AIDPI e da CSC. Todos os alunos assistiram ao vídeo de avaliação do DNPM elaborado pela AIDPI.

Foram analisadas a frequência absoluta e relativa, média e desvio padrão (DP) das variáveis analisadas no questionário aplicado. Conforme orientação do manual da AIDPI, possível atraso foi caracterizado por ausência de um ou mais marcos para a faixa etária da criança. Provável atraso foi caracterizado por ausência de um ou mais marcos da faixa etária anterior à idade da criança ou alteração no perímetro cefálico (PC). Alteração no PC foi definida como PC abaixo do percentil 10 ou acima do percentil 90, na curva do National Center for Health Statistics (NCHS, 2000). Foram comparadas as classificações do desenvolvimento pela AIDPI e pela CSC, calculando-se o coeficiente Kappa. A associação entre atraso do desenvolvimento e as variáveis estudadas foi feita pelo teste de Quiquadrado ou Fisher quando indicado. Foi considerado como nível de significância estatística valor $p$ menor ou igual a 0,05.

Ao final da pesquisa, foi organizada uma oficina denominada Tarde de Lazer, na qual foram convidadas todas as famílias das crianças que participaram da pesquisa e foram abordados os aspectos sociais, emocionais, comportamentais, de linguagem e motor do desenvolvimento infantil nessa faixa etária. Como forma de representação lúdica, foram construídos brinquedos com material reciclado para incentivar o estímulo ao desenvolvimento das crianças. Todos os alunos participaram ativamente do processo de construção do material da pesquisa, da organização da logística da coleta e do planejamento da oficina.

Todas as etapas da pesquisa foram realizadas com a autorização dos pais ou responsáveis pelas crianças por meio da assinatura do Termo de Consentimento Livre e Esclarecido (TCLE). O presente trabalho foi aprovado nos Comitês de Ética em Pesquisa da UFMG e da SMS-BH.

\section{RESULTADOS}

\section{Característica da População}

Foram estudadas 122 crianças de 2 meses a 2 anos de idade moradoras da área de abrangência da Unidade Básica de Saúde (UBS) São Bernardo em Belo Horizonte, Minas Gerais.

A tabela 1 mostra as características da população estudada.

TABELA 1

Características das crianças de 2 a 24 meses da UBS São Bernardo $(n=122)$.

\begin{tabular}{|c|c|c|c|}
\hline Característica & $\begin{array}{c}\text { Presente } \\
(\%)\end{array}$ & $\begin{array}{c}\text { Ausente } \\
(\%)\end{array}$ & $\begin{array}{c}\text { Não relatada } \\
(\%)\end{array}$ \\
\hline $\begin{array}{l}\text { Convivência com } \\
\text { agressividade }\end{array}$ & $19(15,6)$ & $101(82,8)$ & $2(1,6)$ \\
\hline $\begin{array}{l}\text { Convivência com etilismo } \\
\text { ou drogas }\end{array}$ & $18(14,8)$ & $103(84,4)$ & $1(0,8)$ \\
\hline $\begin{array}{l}\text { Convivência com } \\
\text { problemas emocionais }\end{array}$ & $23(18,9)$ & $98(80,3)$ & $1(0,8)$ \\
\hline Frequenta creche & $11(9,0)$ & $111(91,0)$ & 0 \\
\hline $\begin{array}{l}\text { Parente com deficiência } \\
\text { mental }\end{array}$ & $34(27,9)$ & $87(71,3)$ & $1(0,8)$ \\
\hline Histórico de doença grave & $15(12,3)$ & $107(87,7)$ & 0 \\
\hline $\begin{array}{l}\text { Escolaridade da mãe }<8 \\
\text { anos }\end{array}$ & $44(37,0)$ & $72(62,1)$ & $6(4,9)$ \\
\hline Idade da mãe $<20$ anos & $21(17,2)$ & $91(74,6)$ & $10(8,2)$ \\
\hline $\begin{array}{l}\text { Consanguinidade entre pai } \\
\text { e mãe }\end{array}$ & $4(3,3)$ & $118(96,7)$ & 0 \\
\hline Problemas na gestação & $34(27,9)$ & $87(71,3)$ & $1(0,8)$ \\
\hline Problemas durante o parto & $22(18,0)$ & $99(81,1)$ & $1(0,8)$ \\
\hline $\begin{array}{l}\text { Alteração no perímetro } \\
\text { cefálico }\end{array}$ & $13(10,7)$ & $107(87,7)$ & $2(1,6)$ \\
\hline
\end{tabular}


As características com maior frequência na população estudada são a baixa escolaridade das mães, seguida de parentes com deficiência mental e problemas na gestação (hipertensão arterial sistêmica - pré-eclâmpsia em $12,3 \%$; trabalho de parto prematuro em $4,1 \%$; diabetes e infecção do trato urinário em 3,3\%; problemas emocionais em 2,5\% e outras doenças em $5,7 \%$ das mães). Apenas 11 ( $9 \%$ ) crianças frequentavam creche.

A média da idade gestacional foi 38,7 ( $\pm 2,4 \mathrm{DP})$ semanas. Para o peso ao nascimento obteve-se uma média de 3.141,5 ( \pm $526,5)$ gramas, com um valor mínimo de 1.550 e máximo de 4.615 gramas. Quanto à idade gestacional e peso de nascimento, $17(14,0 \%)$ eram prematuras $(<37$ semanas) e $13(10,7 \%)$ apresentaram baixo peso ao nascimento $(<2.500 \mathrm{~g})$.

A maioria das mães acreditava que o desenvolvimento de seus filhos era normal ou adiantado (118/122, 96,7\%). Apenas quatro $(3,3 \%)$ achavam a criança com desenvolvimento atrasado. Não houve associação entre a opinião da mãe e a classificação pela AIDPI $(\mathrm{p}<0,05)$.

\section{Classificação do Desenvolvimento Infantil}

O gráfico 1 apresenta os resultados da avaliação do desenvolvimento neuropscicomotor obtidos por meio da estratégia Atenção às Doenças Prevalentes na Infância (AIDPI).

Verificou-se que, das 122 crianças avaliadas, 95 (77,9\%) apresentaram desenvolvimento normal à observação direta, sendo classificadas como normais ( $\mathrm{n}=20,16,4 \%$ ) ou normais com fator de risco $(n=75,61,5 \%)$.

\section{GrÁfICO 1}

Classificação de crianças entre 2 e 24 meses de idade, segundo critérios da AIDPI ( $\mathrm{n}=122)$. Belo Horizonte, 2009.

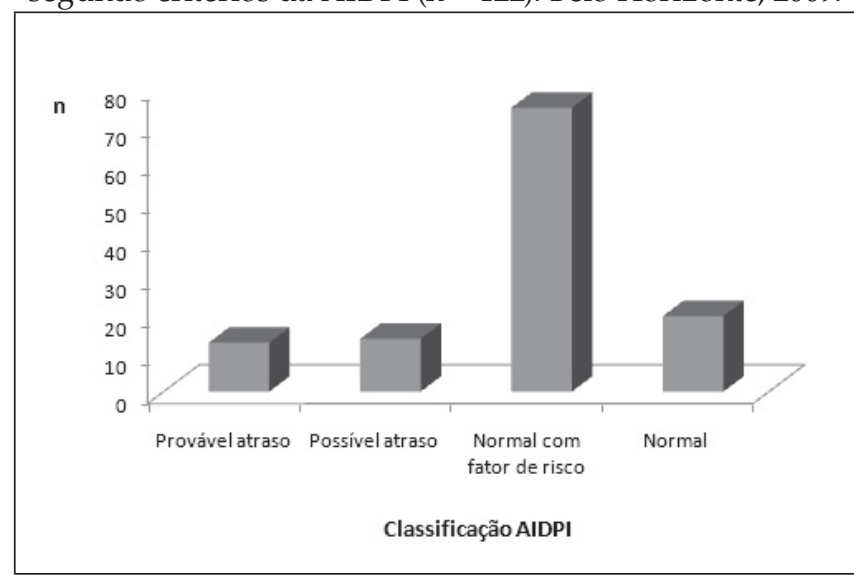

Vinte e sete $(22,1 \%)$ crianças foram classificadas como provável ou possível atraso do desenvolvimento. As crianças que foram classificadas como possível atraso ( $\mathrm{n}=14,11,5 \%$ ) apresentaram ausência de um ou mais marcos de desenvolvimento da AIDPI para a sua faixa etária, mas tinham todos os marcos da faixa etária anterior. Treze $(10,6 \%)$ crianças foram classificadas como provável atraso do desenvolvimento, $\mathrm{e}$ isso ocorreu por verificação de alteração de perímetro cefálico, sendo a grande maioria com PC acima do percentil 90 ( $\mathrm{n}=12$, $9,8 \%$ ). Nenhuma criança apresentou ausência de um ou mais marcos da faixa etária anterior.

Em relação aos marcos de desenvolvimento presentes na Caderneta de Saúde da Criança, observou-se que 64,8\% (n = 79) apresentaram todos os marcos de DNPM esperados para a idade, e 35,2\% (n = 43) apresentaram ausência de um ou mais marcos esperados para a idade. Destas, 37 (30,3\%) tinham os marcos da faixa etária anterior e $6(4,9 \%)$ não tinham os mar$\cos$ da idade anterior.

A tabela 2 mostra a correspondência entre a avaliação pela AIDPI e pela Caderneta de Saúde da Criança (CSC).

TABELA 2

Correspondência entre a avaliação pela AIDPI e pela Caderneta de Saúde da Criança (CSC).

\begin{tabular}{llll}
\hline \multicolumn{1}{c}{ Classificação AIDPI } & \multicolumn{2}{c}{$\begin{array}{c}\text { Classificação da CSC } \\
\text { Normal Atraso }\end{array}$} & Total \\
\hline $\begin{array}{l}\text { Provável atraso } \\
\text { Linha \% }\end{array}$ & 9 & 4 & 13 \\
Possível atraso & 49,2 & 30,8 & 100,0 \\
Linha \% & 28,6 & 10 & 14 \\
Desenvolvimento normal & & 71,4 & 100,0 \\
com fatores de risco & 50 & & \\
Linha \% & 66,7 & 25 & 75 \\
Desenvolvimento normal & 16 & 33,3 & 100,0 \\
Linha \% & 80,0 & 4 & 20 \\
\hline TOTAL & 79 & 20,0 & 100,0 \\
Linha \% & 64,8 & 43 & 122 \\
\hline
\end{tabular}

Ressalta-se que $80 \%$ das crianças com provável atraso pela classificação AIDPI foram consideradas normais pela CSC. A concordância observada entre a classificação pela AIDPI e pela CSC foi de 0,34, coeficiente Kappa igual a - 0,12 ( $p=0,98)$. Ao analisar apenas os dados de observação do desenvolvimento pelos marcos propostos pela AIDPI e pela CSC, a concordância foi 0,70, coeficiente Kappa igual a 0,25 ( $p=0,0005)$.

A tabela 3 descreve os resultados da análise comparativa de alguns fatores de risco para atraso do desenvolvimento e a classificação da AIDPI.

Não houve associação estatisticamente significativa entre as variáveis analisadas e atraso possível/provável do desenvolvimento identificado pela AIDPI. 
TABELA 3

Resultados da análise comparativa de alguns fatores de risco para atraso do desenvolvimento e a classificação da AIDPI

\begin{tabular}{|c|c|c|c|c|c|}
\hline & & $\begin{array}{c}\text { AIDPI } \\
\text { alterado* } \mathrm{n} \\
=27(\%)\end{array}$ & $\begin{array}{c}\text { AIDPI } \\
\text { normal }^{* *} \\
\mathrm{n}=95(\%)\end{array}$ & $\begin{array}{c}\text { OR } \\
(\mathrm{IC} 95 \%)\end{array}$ & $p$ \\
\hline $\begin{array}{l}\text { Frequenta } \\
\text { creche }\end{array}$ & $\begin{array}{l}\text { Sim } \\
\text { Não }\end{array}$ & $\begin{array}{l}4(14,8 \%) \\
23(85,2 \%)\end{array}$ & $\begin{array}{l}7(7,4 \%) \\
88(92,6 \%)\end{array}$ & $\begin{array}{l}2,17 \\
(0,52-8,16)\end{array}$ & 0,43 \\
\hline $\begin{array}{l}\text { Convívio com } \\
\text { problemas } \\
\text { emocionais }\end{array}$ & $\begin{array}{l}\text { Sim } \\
\text { Não }\end{array}$ & $\begin{array}{l}6(22,2 \%) \\
21(77,8 \%)\end{array}$ & $\begin{array}{l}17(17,9 \%) \\
77(81,1 \%)\end{array}$ & $\begin{array}{l}1,29 \\
(0,40-4,08)\end{array}$ & 0,84 \\
\hline $\begin{array}{l}\text { Escolaridade } \\
\text { da mãe }\end{array}$ & $\begin{array}{l}\leq 8 \mathrm{a} \\
>8^{\mathrm{a}}\end{array}$ & $\begin{array}{l}12(46,2 \%) \\
14(53,8 \%)\end{array}$ & $\begin{array}{l}32(35,6 \%) \\
58(64,4 \%)\end{array}$ & $\begin{array}{l}1,55 \\
(0,64-3,76)\end{array}$ & 0,45 \\
\hline $\begin{array}{l}\text { Idade } \\
\text { gestacional }\end{array}$ & $\begin{array}{l}<37 \mathrm{~s} \\
\geq 37 \mathrm{~s}\end{array}$ & $\begin{array}{l}5(19,2 \%) \\
21(80,8 \%)\end{array}$ & $\begin{array}{l}12(12,6 \%) \\
83(87,4 \%)\end{array}$ & $\begin{array}{l}1,65 \\
(0,52-5,19)\end{array}$ & 0,28 \\
\hline $\begin{array}{l}\text { Peso ao } \\
\text { nascer }\end{array}$ & $\begin{array}{l}<2.500 \mathrm{~g} \\
\geq 2.500 \mathrm{~g}\end{array}$ & $\begin{array}{l}3(11,1 \%) \\
24(88,9 \%)\end{array}$ & $\begin{array}{l}10(10,5 \%) \\
85(89,6 \%)\end{array}$ & $\begin{array}{l}1,06 \\
(0,27-4,17)\end{array}$ & 0,58 \\
\hline
\end{tabular}

$\mathrm{a}=$ anos de estudo; $\mathrm{s}=$ semanas; $\mathrm{g}$ = gramas

*possível ou provável atraso ** normal ou normal com fatores de risco

\section{DISCUSSÃO}

O presente estudo confirmou que o instrumento proposto pela AIDIPI é de fácil aplicação e pode ser utilizado por diferentes profissionais atuantes na Atenção Primária, inclusive estudantes da área da saúde. Através da utilização do questionário AIDPI foi possível estabelecer um diagnóstico da situação das crianças de 2 meses a 2 anos de idade em acompanhamento no CSSB e propor uma abordagem para cada situação verificada.

Segundo os resultados, $11,6 \%$ das crianças apresentaram possível atraso do desenvolvimento, e as famílias deveriam ser orientadas sobre a estimulação adequada, como foi feito durante a oficina. As crianças com provável atraso (10,5\%) necessitariam ser referidas para avaliação neuropsicomotora mais detalhada, porém todas apresentavam todos os marcos de desenvolvimento da sua faixa etária. A opção considerada mais adequada pela equipe foi acompanhá-las longitudinalmente com novas medidas do PC e vigilância do DNPM nas consultas médicas de puericultura mensais.

Entre as crianças com desenvolvimento normal, a maioria ( $n=75,61,5 \%$ ) vivia em ambientes com fatores de risco social, como convivência com agressividade, drogas e pessoas com problemas emocionais, refletindo a necessidade de vigilância de sua saúde física e emocional. Como ressaltado no estudo de Pilzs et al., posteriormente, essas crianças podem vir a apresentar transtornos de comportamento ${ }^{11}$.

A comparação entre a avaliação pela AIDPI e pela CSC mostrou que não existe uma boa correlação entre elas (Kappa = -
0,12). A AIDPI estabelece como marcos da faixa etária aqueles que a maioria das crianças deve estar realizando com o objetivo de detectar atrasos e estabelecer condutas. Na CSC, os marcos utilizados são semelhantes aos da AIDPI, porém alguns se situam em faixa etária diferente, mais precoce. Por exemplo, o marco "sentar sem apoio", na CSC, está na faixa etária de 6 a 9 meses de idade e na AIDPI, na faixa de 9 a 12 meses. Talvez isso se dê pelo fato de que o objetivo principal da CSC é orientar a estimulação adequada da criança. Entretanto, como ambos são instrumentos a serem utilizados na Atenção Primária pelos mesmos profissionais, seria importante haver uma maior aproximação entre eles, ou, pelo menos, ser estabelecido quando usar um ou outro.

Apesar disso, os valores encontrados de prevalência de alteração no desenvolvimento, 22,1\% pela AIDPI e 35,2\% pela CSC, são semelhantes ao relatado por outros autores que estudaram crianças brasileiras na mesma faixa etária, utilizando a escala de Denver, com valores entre 21,4 e $27,0 \%{ }^{12,13}$. Ressalte-se que a escala de Denver é um instrumento mais acurado, porém de mais difícil utilização.

Outra observação interessante foi a ausência de correlação entre a opinião materna sobre o desenvolvimento de seu filho e a avaliação com o uso do questionário AIDPI, reforçando a necessidade de observação sistemática do DNPM. Drachler et al. observaram que a percepção materna é mais acurada quando o nível socioeconômico e a escolaridade da mãe são mais elevados. A maior parte da população da área de abrangência do CSSB compreende famílias de baixa renda, o que pode ter contribuído para os resultados encontrados ${ }^{13}$.

Quanto aos fatores de risco para atraso de desenvolvimento, não foram observadas associações significativas, e isso pode ter ocorrido devido ao tamanho amostral. Apesar disso, vale observar que a frequência de atraso foi maior nas crianças que frequentavam creche, o que pode ser um alerta para a qualidade do cuidado relativo à estimulação nesses ambientes. Outros autores também não observaram associação entre atraso de DNPM e escolaridade materna, idade gestacional, peso ao nascimento e frequência à creche $\mathrm{e}^{11,14-16}$.

Apesar do esforço empenhado por toda a equipe do PET-Saúde e dos profissionais do CSSB, algumas famílias não levaram as crianças para as avaliações, mesmo nos dias de campanha de vacinação. É possível que essas crianças constituam um grupo de maior risco de agravo à saúde, inclusive de atraso de DNPM, e isso constitui a principal limitação deste estudo.

Não foram encontrados na revisão da literatura outros estudos que comparassem a avaliação do desenvolvimento com o questionário da AIDPI e da CSC. Outros trabalhos ainda são necessários para contribuir com o aprimoramento do instrumento, especialmente, de acompanhamento prospectivo das crianças. 


\section{CONCLUSÃO}

$\mathrm{O}$ adequado desenvolvimento físico, psíquico e social do ser humano depende, em grande parte, dos cuidados recebidos nos primeiros anos de vida, os quais determinam a capacidade de aprender, de se relacionar e de regular as emoções. Capacitar os profissionais de saúde para a avaliação sistematizada do desenvolvimento infantil, promover ambientes afetivos e com estimulação adequada e envolver a comunidade na discussão deste tema são ações essenciais para se alcançar esse objetivo.

O PET-Saúde, como proposta de integração da educação pelo trabalho, permitiu uma oportunidade rara de convivência e troca de experiências entre alunos e profissionais de diferentes áreas de atuação trabalhando em um projeto comum. A maior parte dos resultados desta experiência não são mensuráveis, e seu impacto na vida profissional e pessoal de todos os atores envolvidos, especialmente os estudantes, contribuirá para uma mudança de paradigma na concepção de saúde e de trabalho em equipe.

\section{REFERÊNCIAS}

1. Brasil. ministério da Saúde. Portal da Saúde [homepage]. PET-Saúde. [acesso em 22 mar. 2010]. Disponível em http:/ / portal.saude.gov.br/portal/saude/profissional/ area.cfm?id_area $=1597$

2. Bispo Júnior JP. Formação em Fisioterapia no Brasil: reflexões sobre a expansão do ensino e os modelos de formação. Hist Ciênc Saúde - Manguinhos. 2009;16(3): 655-68.

3. Universidade Federal de Minas Gerais. Secretaria Municipal de Saúde de Belo Horizonte. Programa de Educação pelo Trabalho em Saúde. Belo Horizonte: UFMG;2009, p. 47.

4. Coelho ZAC, Rezende MB. Atraso no Desenvolvimento. In: Terapia Ocupacional Fundamentação e Prática. Rio de Janeiro: Guanabara Koogan; 2007. p.512-8.

5. Monteiro RCS. Neonatologia In: Terapia Ocupacional Fundamentação e Prática. Rio de Janeiro: Guanabara Koogan; 2007. p. $512-18$

6. Organização Pan-Americana da Saúde. Manual para vigilância do desenvolvimento infantil no contexto da AIDPI. Washington, D.C.: OPAS; 2005.

7. Anderson LM et al. The Effectiveness of Early Childhood Development. Programs. A Systematic Review. Am J Prev Med. 2003;24(3):32-46.

8. Coelho ZAC, Rezende MB. Atraso no Desenvolvimento. In: Terapia Ocupacional Fundamentação e Prática. Rio de Janeiro: Guanabara Koogan; 2007.p. 299-307.

9. Benguigui Y. A estratégia AIDPI como instrumento de mudança do paradigma no ensino da Pediatria. Rev. Pediatr. 2008;9(2):80-3
10. Brasil. Ministério da Saúde. Portal da Saúde [homepage]. Caderneta de Saúde da Criança. 2007 [acesso em 28 mar. 2010]. Disponível em :http://portal.saude.gov.br/portal/ arquivos/pdf/caderneta_crianca_2007.pdf

11. Pilzs EML, Schermann LG. Determinantes biológicos e ambientais no desenvolvimento neuropsicomotor em uma amostra de crianças de Canoas/RS. Ciênc Saúde Coletiva. 2007; 12(1):181-90.

12. Halpern R, Barros AJD, Matijasevich A, Santos IS, Victória CG, Barros FC. Developmental status at age 12 months according to birth weight and family income: a comparison of two Brazilian birth cohorts. Cad Saúde Pública. 2008;24(Supl. 3):444-50.

13. Drachler M L, Aerts DGC, Souza RM, Carvalho LJC, Giugliani EJ, Marshall T. Social inequalities in maternal opinion of child development in southern Brazil. Acta Paediatrica. 2005;94(8):1137-9

14. Santos LM, Santos DN, Bastos ACS, Assis AMO, Prado MS, Barreto ML. Determinants of early cognitive development: hierarchical analysis of a longitudinal study. Cad Saúde Pública. 2008;24(2):427-37.

15. Andrade KC, Souza SB, Szarfarc SC. Desenvolvimento neuromotor e dentição de crianças atendidas em serviços públicos de saúde do Brasil, no primeiro ano de vida. Rev Bras Crescimento Desenvol Humano. 2007;17(2):37-44.

16. Amorim RCA, Laurentino GEC, Barros KMFT, Ferreira ALPR, Moura Filho AG, Raposo MCF. Programa de Saúde da Família: proposta para identificação de fatores de risco para o desenvolvimento neuropsicomotor. Rev Bras Fisioterapia. 2009;13(6):506-13.

\section{CONTRIBUIÇÃO DOS AUTORES}

Todos os autores participaram de todo o processo incluindo elaboração do projeto, revisão bibliográfica, coleta e análise dos dados e redação do artigo.

\section{CONFLITO DE INTERESSES}

Declarou não haver.

\section{ENDEREÇO PARA CORRESPONDÊNCIA}

Cristina Gonçalves Alvim

Departamento de Pediatria - UFMG

Av. Alfredo Balena, 190

Santa Efigênia - Belo Horizonte

CEP. 30130-100 MG

E-mail: cristinagalvim@gmail.com 


\section{ERRATA EM: Revista Brasileira de Educação Médica, volume 36, número 1, suplemento 1, Janeiro-Março 2012.}

ERRATUM IN: Brazilian Journal of Medical Education, volume 36, issue 1, supplement 1, January-March 2012.

Erro de autoria do artigo: "A avaliação do Desenvolvimento Infantil: um Desafio Interdisciplinar", Cristina Gonçalves Alvim, Fabiano Gonçalves Guimarães, Niriana Lara Santos Meinberg, Larissa Tavares Aguiar, Lívia Cristina Guimarães Caetano, Loyane Cabral Carrusca, Luciana Machado Caetano, Ludimila Labanca, Nathália de Magalhães Fonseca, Rafael Antônio Madeira Paulo, Thaysa Leite Tagliaferri, Haliton Alves de Oliveira Junior, Alex Christian da Silva Alves, Aline Zocrato Alves de Sousa. Revista Brasileira de Educação Médica. 2012; 36 (1 supl. 1): 51-6. 